Research Article

\title{
Generalized Weibull-Lindley (GWL) Distribution in Modeling Lifetime Data
}

\author{
Pius Marthin (i) and Gadde Srinivasa Rao \\ Department of Mathematics and Statistics, The University of Dodoma, Dodoma, Tanzania \\ Correspondence should be addressed to Pius Marthin; martinpius01@gmail.com
}

Received 22 May 2020; Revised 1 July 2020; Accepted 22 July 2020; Published 31 August 2020

Academic Editor: Basil K. Papadopoulos

Copyright (C) 2020 Pius Marthin and Gadde Srinivasa Rao. This is an open access article distributed under the Creative Commons Attribution License, which permits unrestricted use, distribution, and reproduction in any medium, provided the original work is properly cited.

In this manuscript, we have derived a new lifetime distribution named generalized Weibull-Lindley (GWL) distribution based on the $T$ - $X$ family of distribution specifically the generalized Weibull- $X$ family of distribution. We derived and investigated the shapes of its probability density function (pdf), hazard rate function, and survival function. Some statistical properties such as quantile function, mode, median, order statistics, Shannon entropy, Galton skewness, and Moors kurtosis have been derived. Parameter estimation was done through maximum likelihood estimation (MLE) method. Monte Carlo simulation was conducted to check the performance of the parameter estimates. For the inference purpose, two real-life datasets were applied and generalized Weibull-Lindley (GWL) distribution appeared to be superior over its competitors including Lindley distribution, Akash distribution, new Weibull-F distribution, Weibull-Lindley (WL) distribution, and two-parameter Lindley (TPL) distribution.

\section{Introduction}

For the past few decades, generation of new distributions has been motivated by the need of fitting complex lifetime data generated from different fields such as engineering, biological and medical sciences, and geology. Probability models are very essential in describing and predicting various real-world problems. Despite the existence of deep literature regarding the development and establishment of new statistical distributions, we are still required to develop new distributions which are more flexible and compatible with the real-world issues to enable generalization [1].

An interesting feature of the new families of generalized distributions is based on their flexibility to model real-life data due to possession of many parameters as compared to most classical distributions. Over 24 new families of generalized distribution have been studied in the literature. The pioneer of this field includes many scholars such as Azzalini [2], Azzalini and Capitania [3], Marshall and Olkin [4], and Gupta et al. [5]. After establishment of the beta generator, defined by Eugene et al. [5] followed by Jones [6], paved the way to the development of many other distributions. Cordeiro and deCastro [7] and Alexander et al. did tremendous work to establish some competing generators which widened the field [1].

Alzaatreh et al. [8] have proposed a link function which uses any probability density function as a generator to generate the $T-X$ families of generalized distributions. They also studied generalized Weibull- $X$ as a special case. Generalized Weibull- $X$ has been proposed by fixing $T$ as a Weibull distribution and $X$ is allowed to take any other form. From the $T-X$ family of distributions, we can establish various generalized distributions by either controlling the distribution of $T$ and varying the forms of $X$ or vice versa.

In this paper, we consider the pdf of Lindley distribution [9] as the form of $X$ distribution. This distribution has more applications in various areas and plays a prominent role in a wide variety of scientific and technological fields such as reliability engineering, survival analysis, advanced semiconductor technologies, actuarial study, and insurance. For modeling some lifetime data, Weibull distribution may not be sufficient, and therefore, distributions with more flexibility to handle the complexity of a real-life system are of 
great demand. The proposed distribution could handle such situations in the fields of science and technology due to the generalizations and modifications of the Weibull distribution to provide more flexibility in modeling complicated real-life problems.

The rest of the manuscript follows the following order: in the subsequent section, we will give the general form of Weibull- $X$ family of distribution due to Alzaatreh et al. [8] and then use one-parameter Lindley distribution [9]. We will introduce the new distribution called generalized Weibull-Lindley (GWL) distribution. We will then derive and discuss the shapes of its pdf, cdf, survival, and hazard functions. In Section 3, we will study some statistical properties of GWL distribution such as the quantile function, the mode, median, order statistics, Shannon entropy, Galton skewness, and Moors kurtosis. We will then estimate the parameters using maximum likelihood method. In Section 4, we will perform a Monte Carlo simulation to check the performance of parameter estimates, and in Section 5, we will apply GWL distribution along with some other distributions to fit two real lifetime datasets. Finally, in Section 6, we provide a general conclusion to our work and some future recommendations. Model fitting will be done using the AdequacyModel package in $\mathrm{R}$ due to Marinho and Bourguignon [10].

\section{Weibull-X Family of Distribution by Alzaatreh et al. [8]}

Let $r(t)$ be the pdf of the random variable $T \epsilon[a, b]$ for $\infty \leq a<b \leq \infty$ and let $W(F(x))$ be the function of the cdf $F(x)$ of any random variable $X$ such that $W(F(x)) \in[a, b]$.

$W(F(x))$ is monotonically and nondecreasing function. $W(F(x)) \longrightarrow a$ as $x \longrightarrow-\infty$ and $W(F(x)) \longrightarrow b$ as $x \longrightarrow \infty$.

From the above, the new distributions were defined as follows:

Let $X$ be the random variable with the pdf $f(x)$ and cdf $F(x)$. Let $T$ be the continuous random variable with $\operatorname{pdf} r(t)$ on $[a, b]$. The cdf of the new distribution is given as $G(x)=\int_{a}^{W(F(x))} r(t) \mathrm{d} t$, where $W(F(x))$ satisfies the conditions written above. The $\operatorname{cdf} G(x)$ can also be written as follows:

$$
G(x)=R[W(F(x))]
$$

where $R(t)$ is the cdf of the random variable $T$.

The corresponding pdf associated with $G(x)$ above is given as

$$
g(x)=\frac{\mathrm{d}}{\mathrm{d} x}[W(F(x))][r[W(F(x))]],
$$

which is the composite function of R.W. $F(x)$. The pdf $r(t)$ is transformed to the new distribution $g(x)$ with the help of the link function $W(F(x))$ which acts as the transformer, and thus, $T$ - $X$ family of distribution name has been provided [1]. When the random variable $X$ is discrete, the resulting family of distribution will also be discrete.

Different forms of $W(F(x))$ will give rise to the new family of distribution. The form of $W(F(x))$ depends on the support of random variable $T$ as defined above.
When the support of $T$ is $a \leq T<\infty$ and if $a \geq 0$ is assumed without loss of generality, then $W(F(x))$ can be defined as $\quad-\log (1-F(x)),(F(x) / 1-F(x))$ $-\log \left(1-F(x)^{\alpha}\right.$ and $\left(F(x)^{\alpha} / 1-F(x)^{\alpha}\right)$.

We know that lifetime distributions have positive domains, and therefore, using the above link functions, we can obtain several types of lifetime distribution families.

Considering Weibull distribution as one of the lifetime distributions, Alzaatreh et al. applied the support of $T$ as $-\log (1-F(x))$. By substituting into equation (1), we obtain the cdf of new family of Weibull distribution as

$$
G(x)=\int_{a}^{-\log (1-F(x))} r(t) \mathrm{d} t=R\{-\log [1-F(x)]\}=R(H(x)),
$$

where $-\log [1-F(x)]$ is the cumulative hazard rate function for the random variable $X$, and here, $R(t)$ is the cdf of the random variable $T$.

The corresponding pdf was given as follows:

$$
\begin{aligned}
g(x) & =\frac{f(x)}{(1-F(x))} r(\{-\log (1-F(x))\}) \\
& =h(x) r(1-F(x))=h(x) r(H(x)),
\end{aligned}
$$

where $h(x)$ is the hazard rate function and $H(x)$ is the cumulative hazard function for the random variable $X$ with cdf $F(x)$.

Now, if the random variable $T$ follows Weibull distribution with parameters $c$ and $\beta$, then the pdf of $T$ will be given as $r(t)=(c / \beta)(t / \beta)^{c-1} \exp \left(-(t / \beta)^{c}\right), t \geq 0,(c, \beta)>0$. From (4), we have the pdf of Weibull- $X$ family as

$$
\begin{aligned}
g(x)= & \frac{c f(x)((1-F(x) / \beta))^{c-1}}{\beta(1-F(x))} \\
& \cdot\left\{\exp -\left[\left(-\frac{\log (1-F(x))}{\beta}\right)\right]^{c}\right\} .
\end{aligned}
$$

Since for the above Weibull distribution the cdf is $R(t)=1-\exp \left(-(t / \beta)^{c}\right)$, then we can write the cdf of Weibull- $X$ as

$$
G(x)=1-\exp \left[-\left\{-\frac{\log (1-F(x))}{\beta}\right\}^{c}\right] .
$$

We now introduce our new distribution as follows. Let the random variable $X$ follows Lindley distribution with parameter $\beta$. According to Lindley (1958) [9], the pdf and cdf will be given as

$$
\begin{aligned}
f(x) & =\frac{\theta^{2}}{(1+\theta)}(1+x) \exp (-\theta x), \quad \text { for } x \geq 0, \theta>0, \\
F(x) & =\int_{0}^{x} \frac{\theta^{2}}{(1+\theta)}(1+t) \exp (-\theta t) \mathrm{d} t \\
& =1-\frac{(\theta+1+\theta x)}{(\theta+1)} \exp (-\theta x) .
\end{aligned}
$$


Survival and hazard functions are given as

$$
\begin{aligned}
& S(x)=\frac{(\theta+1+\theta x)}{(\theta+1)} \exp (-\theta x), \\
& h(x)=\frac{f(x)}{S(x)}=\frac{\theta^{2}(1+x)}{(\theta+1+\theta x)} .
\end{aligned}
$$

From the relation between cumulative hazard and survival functions, we have cumulative hazard function of Lindley distribution as

$$
H(x)=-\ln (S(x))=-\ln \left[\frac{(\theta+1+\theta x)}{(\theta+1)} \exp (-\theta x)\right] .
$$

2.1. The pdf of Generalized Weibull-Lindley (GWL) Distribution. We can now substitute equations (7) and (8) into equation (5) to obtain our new distribution as follows:

$$
\begin{aligned}
g(x)= & \frac{c}{\beta}\left[\frac{\left(\theta^{2}(1+x)\right)}{(1+\theta+\theta x)}\right] \\
& \cdot\left[\left\{\frac{-\log ((\exp (-\theta x)(1+\theta+\theta x)) /(\theta+1))}{\beta}\right\}^{c-1}\right] \\
& \cdot\left\{\exp \left[-\left\{\frac{-\log [((\theta+1+\theta x) \exp (-\theta x)) /(1+\theta)]}{\beta}\right\}\right]\right\}
\end{aligned}
$$

For $x \geq 0,(\theta, \beta, c)>0$ which is the pdf of generalized Weibull-Lindley (GWL) distribution.

Figure 1 describes various shapes of GWL pdf when different parameter sets are considered.

As displayed in Figures 1-3, the pdf of generalized Weibull-Lindley (GWL) distribution has various shapes

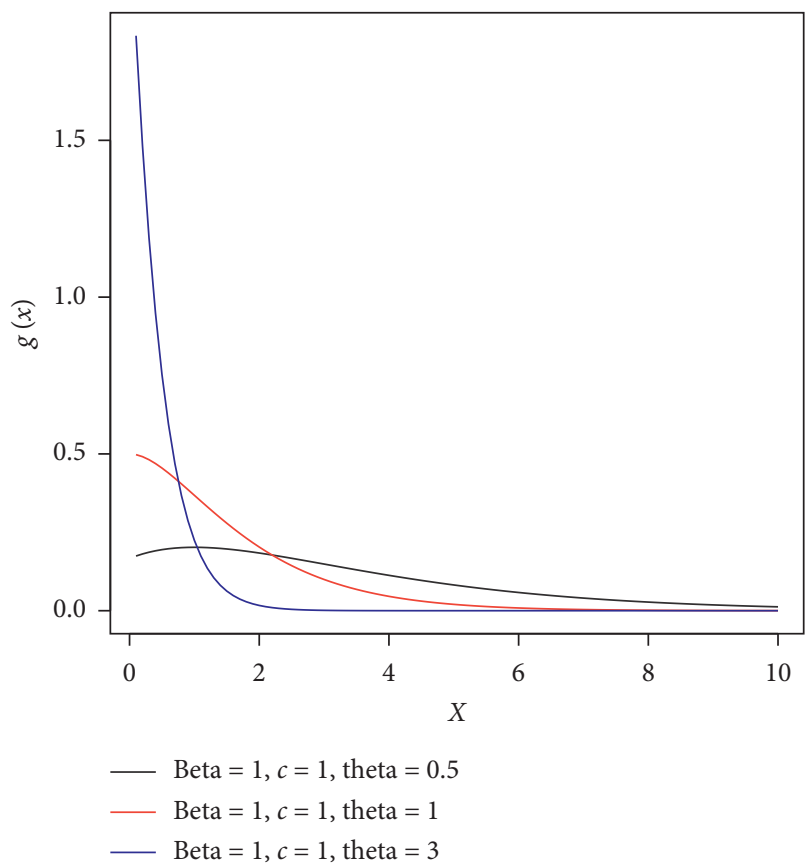

FIGURE 1: The pdf curves for generalized Weibull-Lindley (GWL) distribution for different parameter values.

which can be either increasing or decreasing; it assumes the exponential shape when all parameters are greater or equal to 1 except the case when $\beta=1, c=3$, and $\theta=3$ where the curve rises and declines sharply for lower values of $x$ 's. The pdf also has a bell shape similar to that of normal distribution when $\beta=1, c=3$, and $\theta=0.5$.

2.2. The cdf of Generalized Weibull-Lindley (GWL) Distribution. We can also obtain the cdf by substituting equation (7) into (6) to obtain

$$
G(x)=1-\exp \left[-\left\{-\frac{\log (1-(1-((\theta+1+\theta x) /(\theta+1)) \exp (-\theta \mathrm{x})))}{\beta}\right]^{c}\right]
$$

where $x \geq 0,(\theta, \beta, c)>0$. Figure 2 describes various shapes of generalized Weibull-Lindley (GWL) cumulative distribution function when different sets of parameters are employed.

As seen from Figure 2, generalized Weibull-Lindley (GWL) cumulative distribution function increases to one under different rates when several sets of parameters are considered.
2.3. Survival Function for Generalized Weibull-Lindley (GWL) Distribution. From (12) above, we have the survival function written as a compliment of cdf as

$$
1-G(x)=S(x)=\exp \left[-\left\{-\frac{\log (1-(1-((\theta+1+\theta x) /(\theta+1)) \exp (-\theta \mathrm{x})))}{\beta}\right\}^{c}\right]
$$

Figure 3 displays survival distribution for generalized Weibull-Lindley (GWL) distribution when different sets of parameters are considered.
As displayed in Figure 3, we observe multiple shapes of survival functions which can either decline sharply at lower values of $x$ 's or stay constant when $c=3, \beta=2$, and $\theta=3$, 


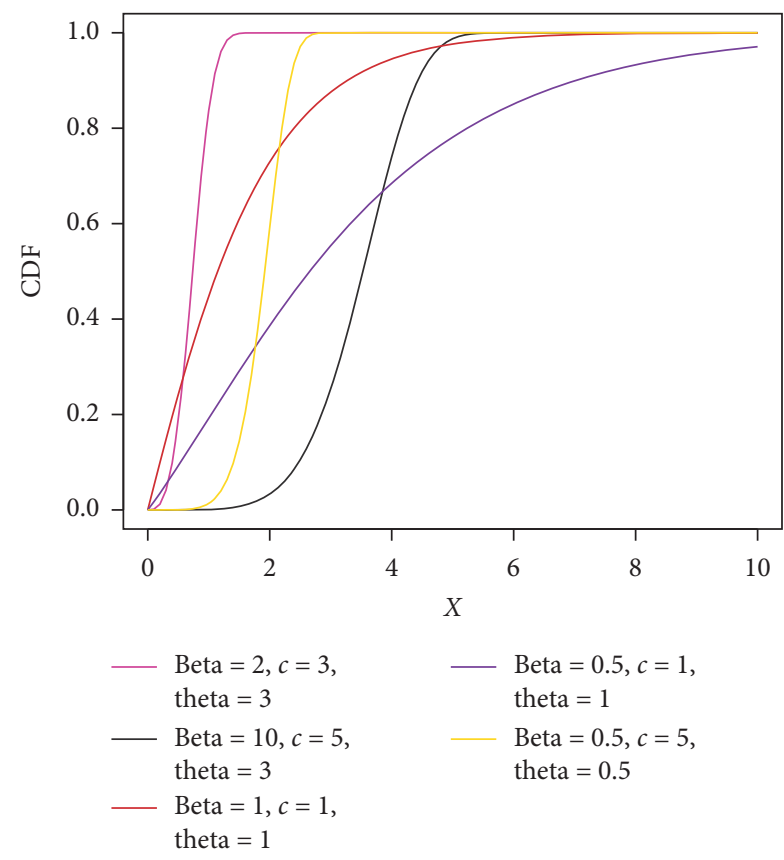

FIgURE 2: The cdf curves for generalized Weibull-Lindley (GWL) distribution for different parameter values.

we also have constant survival at earlier time with sharp decline and then constant when $c=5, \beta=10$, and $\theta=3$, we have an exponential decline of survivals when $c=1, \beta=1$, and $\theta=1$ and $c=1, \beta=0.5$, and $\theta=1$. These properties may be useful in modeling real lifetime data coming from a complicated system or process.

\subsection{Hazard Function for Generalized Weibull-Lindley (GWL)} Distribution. The hazard function will be given using the pdf and survival function in equations (13) and (11):

$$
\begin{aligned}
h(x)= & \frac{g(x)}{S(x)}=\frac{c}{\beta}\left[\frac{\left(\theta^{2}(1+x)\right)}{(1+\theta+\theta x)}\right] \\
& \cdot\left[\left\{\frac{-\log ((\exp (-\theta x)(1+\theta+\theta x)) /(\theta+1))}{\beta}\right\}^{c-1}\right] .
\end{aligned}
$$

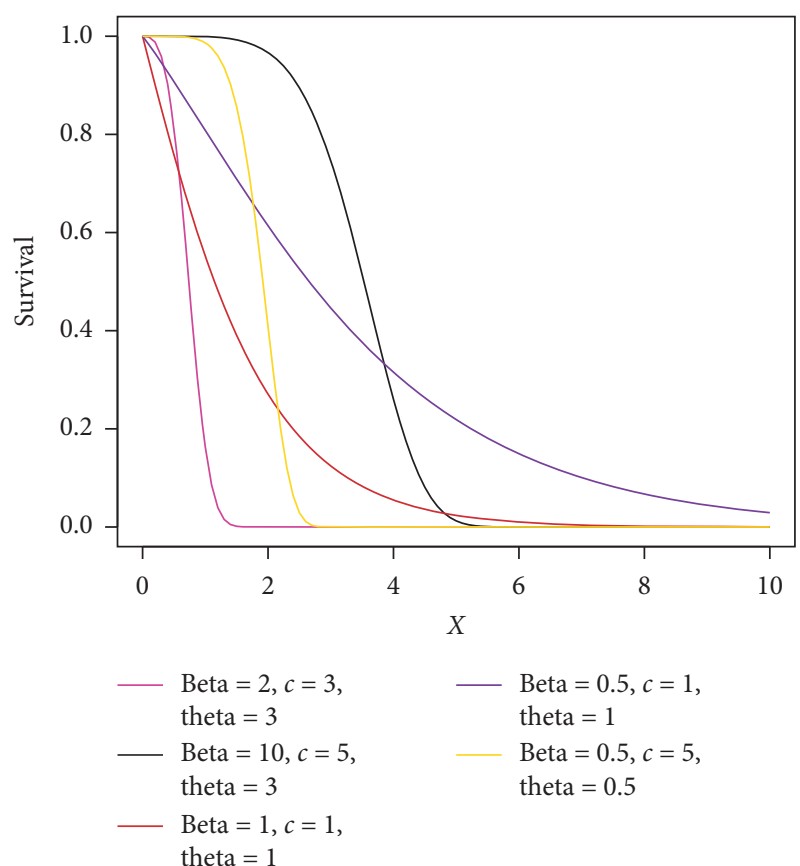

FIGURE 3: Survival curves for generalized Weibull-Lindley (GWL) distribution for different parameter values.

Figure 4 displays various shapes of hazard function for generalized Weibull-Lindley (GWL) distribution when different sets of parameters are considered.

From Figure 4, we can see that the generalized Weibull-Lindley (GWL) distribution has multiple shapes for hazard function which can be either constantly increasing when ( $c=1, \beta=1, \theta=1)$, exponentially increasing when $c=3, \beta=5$, and $\theta=2$, exponentially decreasing when $(c=3, \beta=15, \theta=0.5)$, or "bath tub" shape when $c=10, \beta=1$, and $\theta=0.09$. Similar to the survival curves, various shapes for the hazard function provide wide coverage ground in modeling a complex lifetime dataset.

2.5. The Cumulative Hazard Function for Generalized Weibull-Lindley (GWL) Distribution. From the survival function, we can directly obtain the cumulative hazard function of the GWL like as given below:

$$
H(x)=-\ln (S(x))=-\ln \left(\exp \left[-\left\{-\frac{\log (1-(1-((\theta+1+\theta x) /(\theta+1)) \exp (-\theta \mathrm{x})))}{\beta}\right\}^{c}\right]\right)
$$

which is simplified as follows:

$$
H(x)=-\left\{-\frac{\log (1-(1-((\theta+1+\theta x) /(\theta+1)) p \exp (-\theta x)))}{\beta}\right\}^{c}
$$




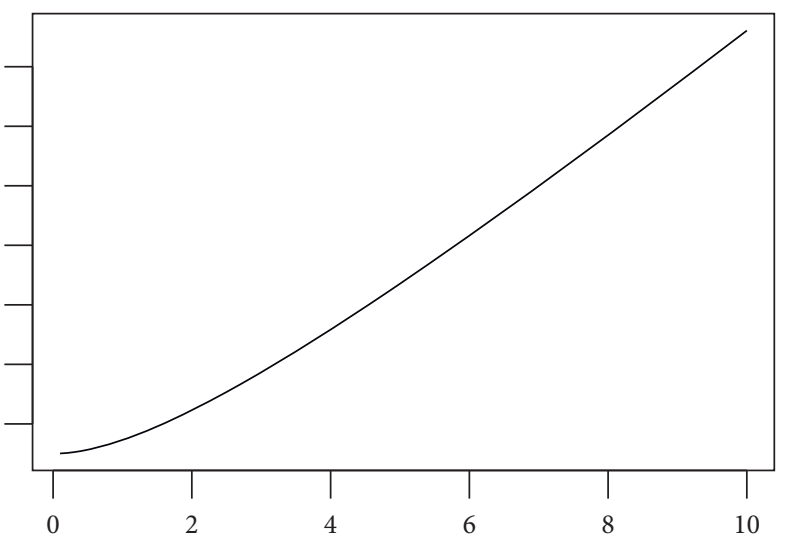

$c=1$, beta $=1$, theta $=1$

(a)

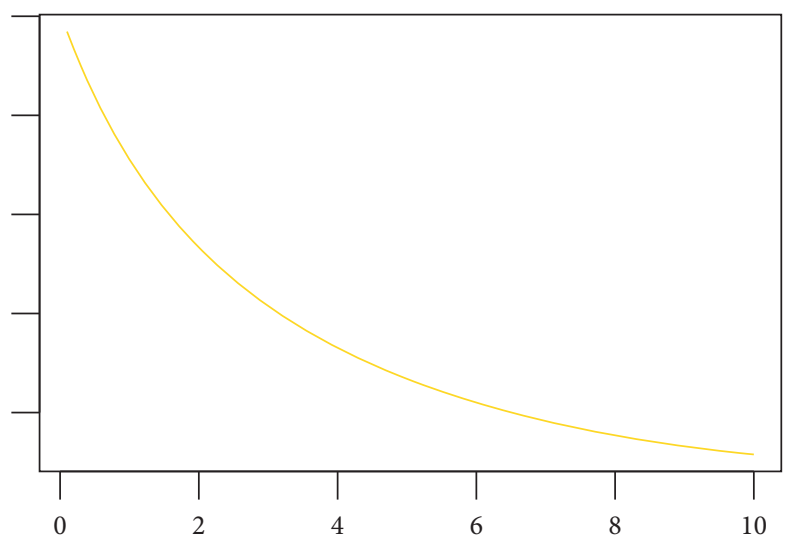

$c=3$, beta $=15$, theta $=0.5$

(c)

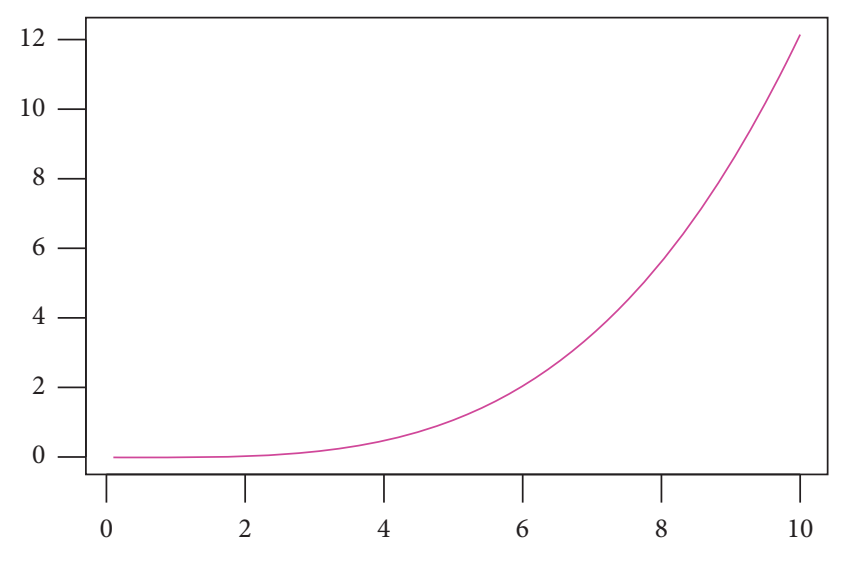

(b)

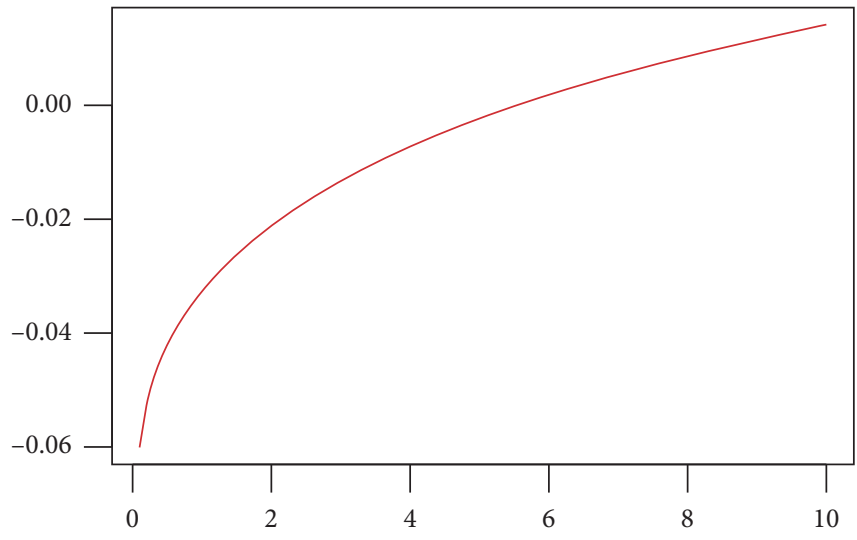

- $c=0.1$, beta $=15$, theta $=3$

(d)

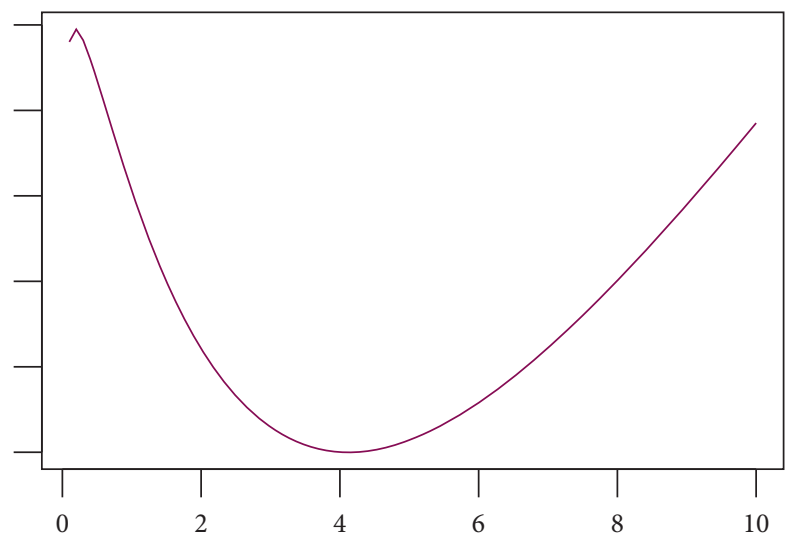

$-c=10$, beta $=1$, theta $=0.09$

(e)

Figure 4: Hazard curves for generalized Weibull-Lindley (GWL) distribution for different parameter values. 


\section{Some Statistical Properties of Generalized Weibull-Lindley (GWL) Distribution}

Here, we will derive and discuss some statistical properties of GWL distribution including quantile function, Galton skewness, Moors kurtosis, mode, median, Shannon entropy, and order statistics.
3.1. The Quantile Function of Generalized Weibull-Lindley (GWL) Distribution. From the cdf equation of generalized Weibull-Lindley, we can obtain the quantile function through inversion like as follows:

$$
\begin{aligned}
G(x) & =1-\exp \left[-\left\{-\frac{\log (1-(1-((\theta+1+\theta x) /(\theta+1)) \exp (-\theta \mathrm{x})))}{\beta}\right]^{c}\right], \\
y & =G(x)=1-\exp \left[-\left\{-\frac{\log (1-(1-((\theta+1+\theta x) /(\theta+1)) \exp (-\theta x)))}{\beta}\right\}^{c}\right], \\
y & =1-\exp \left[-\left\{-\frac{\log (1-F(x))}{\beta}\right\}^{c}\right] .
\end{aligned}
$$

The above can be written as

$$
\begin{aligned}
x-1 & =\exp \left[-\left\{-\frac{\log (1-F(y)}{\beta}\right\}^{c}\right], \\
\log (x-1) & =\left[-\left\{-\frac{\log (1-F(y))}{\beta}\right\}^{c}\right] \\
\beta^{c} \log (1-x) & =-[-\log (1-F(y))]^{c}, \\
-\beta \log (1-x)^{(1 / c)} & =-\log [1-F(y)], \\
F(y) & =1-\exp \left\{\left[\beta(1-x)^{(1 / c)}\right]\right\}, \\
y & =F^{-1}\left[1-\exp \left\{-\beta(-\ln (1-x))^{(1 / c)}\right\}\right] .
\end{aligned}
$$

We know that $F(y)$ is the cdf of Lindley distribution whose quantile is given as

$$
1-\frac{1}{\theta}-\frac{1}{\theta} W_{-1}[(1+\theta)(p-1) \exp (-1-\theta)] .
$$

Therefore, the quantile function of generalized Weibull-Lindley distribution can be written as

$$
\begin{aligned}
Q_{p}= & 1-\frac{1}{\theta}-\frac{1}{\theta} W_{-1}\left[( 1 + \theta ) \left\{1-1-\exp \left\{\left\{-\beta(-\ln (1-x))^{(1 / c)}\right\}\right\}\right.\right. \\
& \cdot \exp (-1-\theta)]
\end{aligned}
$$

which is simplified as follows:

$$
\begin{aligned}
Q_{p}= & -1-\frac{1}{\theta}-\frac{1}{\theta} W_{-1}\left[( 1 + \theta ) \left\{-\exp \left\{-\beta(-\ln (1-x))^{(1 / c)}\right\}\right.\right. \\
& \cdot \exp (-1-\theta)]
\end{aligned}
$$

where $(\beta, \theta, c)>0$, and $W_{-1}$ is the negative branch of the Lambert $W$ function.

3.2. The Median of Generalized Weibull-Lindley (GWL) Distribution. We can obtain the median by solving the following equation:

$$
Q_{p}=-1-\frac{1}{\theta}-\frac{1}{\theta} W_{-1}\left[(1+\theta)\left\{-\exp \left\{-\beta(-\ln (1-x))^{(1 / c)}\right\} \exp (-1-\theta)\right]=0.5\right.
$$

For specified values of the parameter, we can obtain the median of this distribution although the computations will be tedious due to the presence of complicated Lambert function.

3.3. Skewness and Kurtosis. Like in many generalized families of distributions, moments of generalized Weibull-Lindley (GWL) is quite complicated to obtain since it involves the expansion of the pdf. In this case, we suggest the use of Moors kurtosis and Galton skewness which use quantile functions $[11,12]$ :

$$
\begin{aligned}
& \text { Galton skewness } S=\frac{Q_{(6 / 8)}-2 Q_{(4 / 8)}+Q_{(2 / 8)}}{Q_{(6 / 8)}+Q_{(2 / 8)}}, \\
& \text { Moors kurtosis } K=\frac{Q_{(7 / 8)}-2 Q_{(5 / 8)}+Q_{(3 / 8)}-Q_{(1 / 8)}}{Q_{(6 / 8)}+Q_{(2 / 8)}} .
\end{aligned}
$$

We have computed both quantities for different sets of parameters and results are displayed in Table 1. From Table 1, we can see that the generalized Weibull-Lindley (GWL) is a positively skewed distribution. Further, GWL has 
TABLE 1: Moors kurtosis and Galton skewness for GWL distribution at different parameter combinations.

\begin{tabular}{|c|c|c|c|c|}
\hline$\beta$ & $c$ & $\theta$ & Galton skewness & Moors kurtosis \\
\hline 5 & 5 & 1 & 7.00 & -0.0480 \\
\hline 4 & 4 & 1 & 3.33 & -0.0320 \\
\hline 3 & 3 & 1 & 4.22 & -0.0062 \\
\hline 2 & 2 & 1 & 2.85 & -0.0440 \\
\hline 1 & 1 & 1 & 1.65 & 0.1870 \\
\hline 5 & 5 & 2 & 7.00 & -0.0485 \\
\hline 4 & 4 & 2 & 3.33 & -0.0323 \\
\hline 3 & 3 & 2 & 4.22 & -0.0062 \\
\hline 2 & 2 & 2 & 2.85 & 0.0441 \\
\hline 1 & 1 & 2 & 1.65 & 0.1870 \\
\hline 5 & 5 & 3 & 7.00 & -0.0484 \\
\hline 4 & 4 & 3 & 3.33 & -0.0323 \\
\hline 3 & 3 & 3 & 4.22 & -0.0062 \\
\hline 2 & 2 & 3 & 2.85 & 0.0441 \\
\hline 1 & 1 & 3 & 1.65 & 0.1870 \\
\hline 5 & 5 & 4 & 7.00 & -0.0484 \\
\hline 4 & 4 & 4 & 3.33 & -0.0323 \\
\hline 3 & 3 & 4 & 4.22 & -0.0061 \\
\hline 2 & 2 & 4 & 2.85 & 0.0441 \\
\hline 1 & 1 & 4 & 1.65 & 0.1870 \\
\hline 5 & 5 & 5 & 7.00 & -0.0484 \\
\hline 4 & 4 & 5 & 3.33 & -0.0320 \\
\hline 3 & 3 & 5 & 4.22 & -0.0062 \\
\hline 2 & 2 & 5 & 2.85 & 0.0442 \\
\hline 1 & 1 & 5 & 1.65 & 0.1870 \\
\hline
\end{tabular}

a normal approximation with lower values of parameter combinations (beta and $c$ ) while highly skewed to the right with higher values of parameter combinations. On the other hand, we observe high values of Moors kurtosis with the decrease in parameter values.

For better visualization of the above properties, respective mesh plots were obtained. We also employed a bivariate standard Gaussian distribution as a reference. Figure 5 displays the skewness and kurtosis of the GWL distribution with respect to a standard normal distribution.
From Figure 5, we see that as compared to standard Gaussian distribution, GWL distribution is skewed to the right with larger values of parameter combinations. We also observe spikes due to various shapes of its pdf when different parameter combinations are used. These properties may be suitable to model lifetime data of a complicated real-life system.

3.4. The Mode of Generalized Weibull-Lindley Distribution. From the pdf

$$
g(x)=\frac{c}{\beta}\left[\frac{\left(\theta^{2}(1+x)\right)}{(1+\theta+\theta x)}\right]\left[\left\{\frac{-\log (\exp (-\theta x)(1+\theta+\theta x) /(\theta+1))}{\beta}\right\}^{c-1}\right]\left\{\exp \left[-\left\{\frac{-\log [(\theta+1+\theta x) \exp (-\theta x) /(1+\theta)]}{\beta}\right\}^{c}\right]\right\}
$$

We can obtain the mode of this distribution by solving the equation $(\mathrm{d} / \mathrm{d} x)(g(x))=0$.
For simplification, apply natural logarithm both sides, and we have

$$
\begin{aligned}
\ln (g(x))= & \ln (c)+\ln \left(\theta^{2}\right)+\ln (1+x)-\ln (\beta)-\ln (\theta+1+\theta x)+\ln \left(-\left(\log (1-(1-((\theta+1+\theta x) /(\theta+1)) \exp (-\theta x) / \beta))^{c-1}\right)\right) \\
& -\ln \left(1-\left(1-\frac{(\theta+1+\theta x)}{(\theta+1)} \exp (-\theta x)\right)\right)+\left[-\frac{\log (1-(1-((\theta+1+\theta x) /(\theta+1)) \exp (-\theta x)))}{\beta}\right]^{c} .
\end{aligned}
$$


Galton skewness in function of theta, beta, and $c$

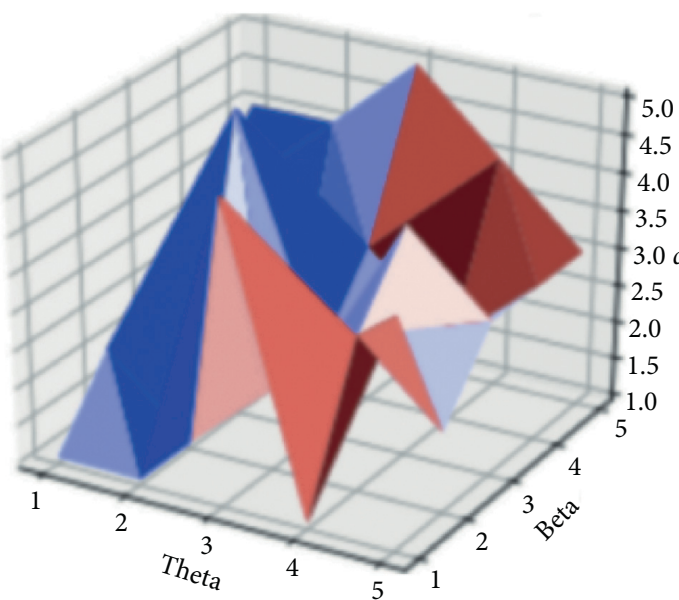

(a)

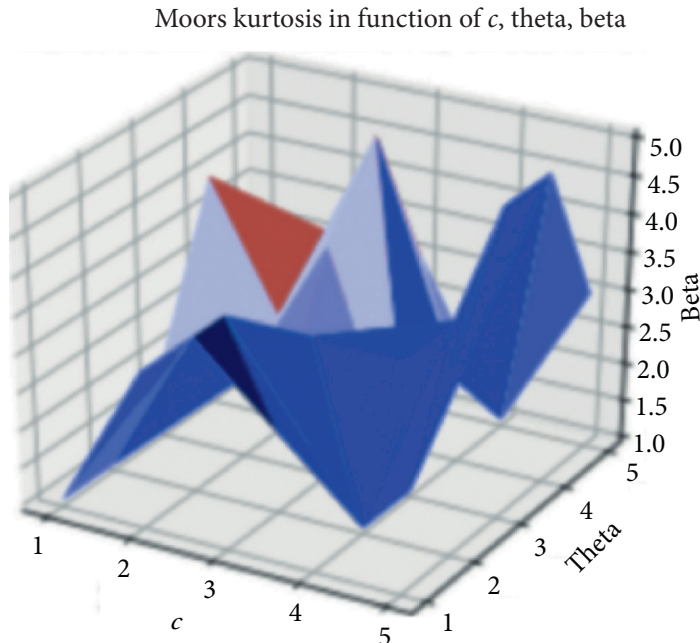

(c)
Standard Gaussian distribution

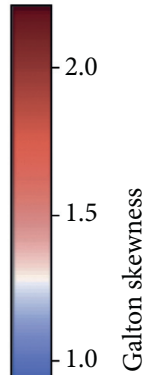

$-0.5$

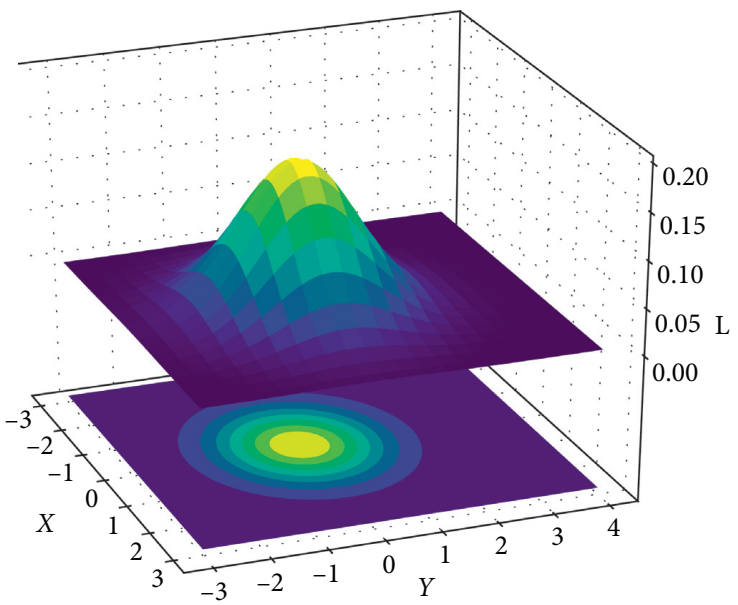

(b)

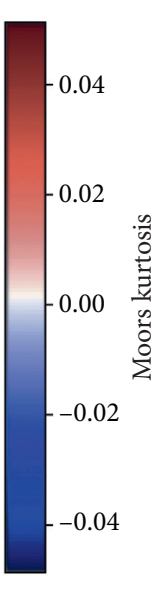

Standard Gaussian distribution

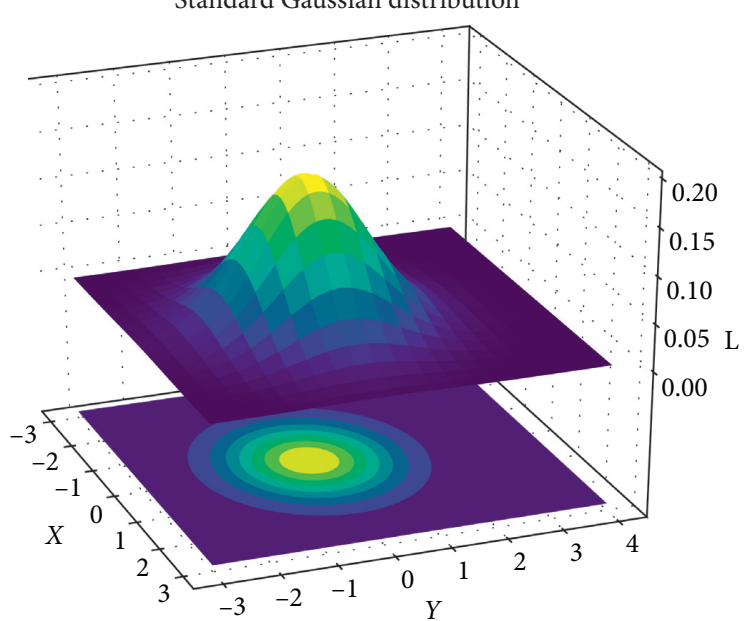

(d)

Figure 5: Galton skewness and Moors kurtosis of GWL for different sets of parameter combination.

By taking derivative with respect to $x$ both sides of the above equation and simplifying, we obtain

$$
\begin{aligned}
\frac{d}{d x}(g(x))= & \left\{\frac{1}{(1+x)}-\frac{\theta}{(\theta+1+x)}-\frac{(c+1)}{\log [((\theta+1+\theta x) /(\theta+1)) \exp (-\theta x)]}\left\{\frac{-\theta}{(\theta+1+\theta x)}+\theta\right\}\right. \\
& \left.+\frac{(c-1)}{x}-\left(\frac{\theta}{(1+\theta+\theta x)}-\theta\right)\left(\frac{c}{\beta}\right)\left\{\frac{-1}{\beta^{c}}\left[-\frac{(\theta+1+\theta x)}{(\theta+1)} \exp (-\theta x)\right]^{c}\right\}^{1-\frac{1}{c}}\right\} g(x) .
\end{aligned}
$$


Therefore, we can obtain the mode of generalized Weibull-Lindley (GWL) distribution by solving the following equation:

$$
\begin{aligned}
& \left\{\frac{1}{(1+x)}-\frac{\theta}{(\theta+1+\theta x)}-\frac{(c+1)}{-\log [((\theta+1+\theta x) /(\theta+1)) \exp (-\theta x)]}\left\{\frac{-\theta}{(\theta+1+\theta x)}+\theta\right\}\right. \\
& \left.\quad+\frac{(c-1)}{x}-\left(\frac{\theta}{(1+\theta+\theta x)}-\theta\right)\left(\frac{c}{\beta}\right)\left\{\frac{-1}{\beta^{c}}[-((\theta+1+\theta x) /(\theta+1)) \exp (-\theta x)]^{c}\right\}^{1-\frac{1}{c}}\right\} g(x)=0 .
\end{aligned}
$$

3.5. Shannon Entropy of Generalized Weibull-Lindley (GWL) Distribution. As a measure of variation of uncertainty, Alzaatreh et al. [8] have given the general formula of obtaining Shannon entropy of a random variable $X$ which follows the Weibull- $X$ family of distribution with pdf:

$$
g(x)=\frac{f(x)}{(1-F(x))} r(\{-\log 1-F(x)\})
$$

as

$$
\eta_{X}=-E\left[\log f\left\{F^{-1}\left(1-e^{-T}\right)\right\}\right]-\mu_{T}+\eta_{T},
$$

where $\mu_{T}$ and $\eta_{T}$ are the mean and Shannon entropy of the random variable $T$ with pdf $r(t)$.

For the case of generalized Weibull- $X$, we obtain Shannon entropy by substituting mean and Shannon entropy of the Weibull distribution in the above equation:

$$
\begin{aligned}
\eta_{X}=- & E\left[\log f\left\{F^{-1}\left(1-e^{-T}\right)\right\}\right]-\beta \Gamma\left(1+\frac{1}{c}\right) \\
& +\gamma\left(1-\frac{1}{c}\right)-\log \left(\frac{c}{\beta}\right)+1,
\end{aligned}
$$

where $c$ and $\beta$ are the parameters of Weibull distribution, and for our case, $F($.$) and f($.$) will represent the cdf and pdf$ of Lindley distribution.

From the previous equations, we had the $\operatorname{pd} f$ and $c \mathrm{~d} f$ of Lindley distribution as

$$
\begin{aligned}
& f(x)=\frac{\theta^{2}}{(1+\theta)}(1+x) \exp (-\theta x), \\
& F(x)=1-\frac{(\theta+1+\theta x)}{(\theta+1)} \exp (-\theta x) \quad \text { for } x \geq 0, \theta>0 .
\end{aligned}
$$

The inverse of $F(x)$ is given as

$$
F^{-1}(x)=-1-\frac{1}{\theta}-\frac{1}{\theta} W_{-1}\{(1+\theta)(p-1) \exp (-1-\theta)\} \text {. }
$$

where $W_{-1}$ is the lower part of the Lambert $W$ function.

Therefore,

$$
\begin{aligned}
F^{-1}\left(1-e^{-T}\right)= & -1-\frac{1}{\theta}-\frac{1}{\theta} W_{-1}\left\{(1+\theta)\left(-e^{-T}\right) \exp (-1-\theta)\right\}, \\
f\left\{F^{-1}\left(1-e^{-T}\right)\right\}= & \frac{\theta^{2}}{(1+\theta)}\left(1+\left[-1-\frac{1}{\theta}-\frac{1}{\theta} W_{-1}\left\{(1+\theta)\left(-e^{-T}\right) \exp (-1-\theta)\right\}\right]\right) \\
& \cdot \exp \left(-\theta\left[-1-\frac{1}{\theta}-\frac{1}{\theta} W_{-1}\left\{(1+\theta)\left(-e^{-T}\right) \exp (-1-\theta)\right\}\right]\right) .
\end{aligned}
$$

This gives

$$
\begin{aligned}
\log \left(f\left\{F^{-1}\left(1-e^{-T}\right)\right\}\right)= & 2 \log (\theta)-\log (1+\theta)+\log \left(1+\left[-1-\frac{1}{\theta}-\frac{1}{\theta} W_{-1}\left\{(1+\theta)\left(-e^{-T}\right) \exp (-1-\theta)\right\}\right]\right) \\
& +\left(-\theta\left[-1-\frac{1}{\theta}-\frac{1}{\theta} W_{-1}\left\{(1+\theta)\left(-e^{-T}\right) \exp (-1-\theta)\right\}\right]\right) .
\end{aligned}
$$


From the above equation, we can obtain the expected value of $\log \left[f\left\{F^{-1}\left(1-e^{-T}\right)\right\}\right]$ as

$$
\begin{aligned}
& E\left\{2 \log (\theta)-\log (1+\theta)+\log \left(1+\left[-1-\frac{1}{\theta}-\frac{1}{\theta} W_{-1}\left\{(1+\theta)\left(-e^{-T}\right) \exp (-1-\theta)\right\}\right]\right)\right. \\
& \left.+\left(-\theta\left[-1-\frac{1}{\theta}-\frac{1}{\theta} W_{-1}\left\{(1+\theta)\left(-e^{-T}\right) \exp (-1-\theta)\right\}\right]\right)\right\} .
\end{aligned}
$$

And hence, we have the Shannon entropy of generalized Weibull-Lindley (GWL) distribution given as

$$
\begin{aligned}
\eta_{X}= & -E\left\{2 \log (\theta)-\log (1+\theta)+\log \left(1+\left[-1-\frac{1}{\theta}-\frac{1}{\theta} W_{-1}\left\{(1+\theta)\left(-e^{-T}\right) \exp (-1-\theta)\right\}\right]\right)\right. \\
& \left.+\left(-\theta\left[-1-\frac{1}{\theta}-\frac{1}{\theta} W_{-1}\left\{(1+\theta)\left(-e^{-T}\right) \exp (-1-\theta)\right\}\right]\right)\right\}-\beta \Gamma\left(1+\frac{1}{c}\right)+\gamma\left(1-\frac{1}{c}\right)-\log \left(\frac{c}{\beta}\right)+1 \text { for }(\beta, \theta, c)>0
\end{aligned}
$$

3.6. Order Statistic for Generalized Weibull-Lindley (GWL) Distribution. Order statistic of the random variable has multiple applications such as modeling extreme datasets, estimations of various statistical quantities, and many more.
We can define a distribution of an order statistic for the generalized Weibull-Lindley distribution like as follows:

Let us assume we have $X_{1}, X_{2}, X_{3}, \ldots, X_{n}$ be iid generalized Weibull-Lindley (GWL) random variables with pdf given as

$$
g(x)=\frac{c}{\beta}\left[\frac{\left(\theta^{2}(1+x)\right)}{(1+\theta+\theta x)}\right]\left[\left\{\frac{-\log (\exp (-\theta x)(1+\theta+\theta x) /(\theta+1))}{\beta}\right\}^{c-1}\right]\left\{\exp \left[-\left\{\frac{-\log [(\theta+1+\theta x) \exp (-\theta x) /(1+\theta)]}{\beta}\right\}^{c}\right]\right\}
$$

and cdf given as

$$
G(x)=1-\exp \left[-\left\{-\frac{\log (1-(1-((\theta+1+\theta x) /(\theta+1)) \exp (-\theta x)))}{\beta}\right\}\right]^{c} .
$$

Let $X_{(1)}, X_{(2)}, X_{(3)}, \ldots, X_{(n)}$ be $n$-order statistics for the above random variables. The $r^{\text {th }}$ order statistic has the pdf given as follows:

$$
f\left(x_{r}\right)=\frac{n !}{(r-1) !(n-r) !} \sum_{i=1}^{n-r}(-1)^{i}\left(\begin{array}{c}
n-r \\
i
\end{array}\right) g(x)(G(x))^{(i+r-1)},
$$

where $G(x)$ and $g(x)$ are the cdf and pdf of any continuous distribution in our case being generalized Weibull-Lindley distribution.

We can now write the pdf of $r^{\text {th }}$ order statistic for generalized Weibull-Lindley as

$$
\begin{aligned}
f\left(x_{r}\right)= & \frac{n !}{(r-1) !(n-r) !} \sum_{i=1}^{n-r}(-1)^{i}\left(\begin{array}{c}
n-r \\
i
\end{array}\right) \frac{c}{\beta}\left[\frac{\left(\theta^{2}(1+x)\right)}{(1+\theta+\theta x)}\right]\left[\left\{\frac{-\log (\exp (-\theta x)(1+\theta+\theta x) /(\theta+1))}{\beta}\right\}^{c-1}\right] \\
& \cdot \exp \left[-\left\{\frac{-\log [(\theta+1+\theta x) \exp (-\theta x) /(1+\theta)]}{\beta}\right\}^{c}\right]\left\{1-\exp \left[-\left\{-\frac{\log (1-(1-((\theta+1+\theta x) /(\theta+1)) \exp (-\theta \mathrm{x})))}{\beta}\right\}^{c}\right]\right\}^{(i+r-1)}
\end{aligned}
$$


Now, the smallest $1^{\text {st }}$ order statistic has the pdf given as

$$
\begin{aligned}
f\left(x_{1}\right)= & n \sum_{i=1}^{n-1}(-1)^{i}\left(\begin{array}{c}
n-1 \\
i
\end{array}\right) \frac{c}{\beta}\left[\frac{\left(\theta^{2}(1+x)\right)}{(1+\theta+\theta x)}\right]\left[\left\{\frac{-\log (\exp (-\theta x)(1+\theta+\theta x) /(\theta+1))}{\beta}\right\}^{c-1}\right] \\
& \cdot\left\{\exp \left[-\left\{\frac{-\log [(\theta+1+\theta x) \exp (-\theta x) /(1+\theta)]}{\beta}\right\}^{c}\right]\right\} \\
& \cdot\left\{\exp \left\{1-\exp \left[-\left\{-\frac{\log (1-(1-((\theta+1+\theta x) /(\theta+1)) \exp (-\theta x)))}{\beta}\right\}^{c}\right]\right\} \exp \left[-\left\{-\frac{\log (1-(1-((\theta+1+\theta x) /(\theta+1)) \exp (-\theta x)))}{\beta}\right\}^{c}\right]\right\}^{(i)} .
\end{aligned}
$$

We also have the pdf of the largest $n^{\text {th }}$ order statistic written as

$$
\begin{aligned}
f\left(x_{n}\right)= & n \frac{c}{\beta}\left[\frac{\left(\theta^{2}(1+x)\right)}{(1+\theta+\theta x)}\right]\left[\left\{\frac{-\log (\exp (-\theta x)(1+\theta+\theta x) /(\theta+1))}{\beta}\right\}^{c-1}\right] \exp \left[-\left\{\frac{-\log [(\theta+1+\theta x) \exp (-\theta x) /(1+\theta)]}{\beta}\right\}^{c}\right] \\
& \cdot\left\{1-\exp \left[-\left\{-\frac{\log (1-(1-((\theta+1+\theta x) /(\theta+1)) \exp (-\theta x)))}{\beta}\right\}^{c}\right]\right\}^{(n-1)} .
\end{aligned}
$$

Therefore, the extremes pdf for generalized Weibull-Lindley are given in the above equations.

3.7. Parameter Estimation through Maximum Likelihood Method. To employ the maximum likelihood, we need to establish the likelihood function of the sample observations.
This is the probability of observing a particular set of data given the chosen probability distribution model for the data [13].

Let us assume we have $X_{1}, X_{2}, X_{3}, \ldots, X_{n}$ as random sample of size " $n$ " drawn from the generalized Weibull-Lindley (GWL) distribution with pdf given as

$$
g(x)=\frac{c}{\beta}\left[\frac{\left(\theta^{2}(1+x)\right)}{(1+\theta+\theta x)}\right]\left[\left\{\frac{-\log (\exp (-\theta x)(1+\theta+\theta x) /(\theta+1))}{\beta}\right\}^{c-1}\right]\left\{\exp \left[-\left\{\frac{-\log [(\theta+1+\theta x) \exp (-\theta x) /(1+\theta)]}{\beta}\right\}^{c}\right]\right\}
$$

The likelihood function can be written as

$$
\begin{aligned}
L(x, \psi)= & \prod_{i=1}^{n}\left\{\left\{\frac{c}{\beta}\left[\frac{\left(\theta^{2}\left(1+x_{i}\right)\right)}{\left(1+\theta+\theta x_{i}\right)}\right]\left[\left\{\frac{-\log \left(\exp \left(-\theta \mathrm{x}_{\mathrm{i}}\right)\left(1+\theta+\theta x_{i}\right) /(\theta+1)\right)}{\beta}\right\}^{c-1}\right]\right.\right. \\
& \left.\left.\cdot\left\{\exp \left[-\left\{\frac{-\log \left[\left(\theta+1+\theta x_{i}\right) \exp \left(-\theta x_{i}\right) /(1+\theta)\right]}{\beta}\right\}\right]\right\}\right\}\right\},
\end{aligned}
$$


where $\psi=(\theta, \beta, c)$.

The log-likelihood function can be written as

$$
\begin{aligned}
l(x, \psi)= & 2 n \ln (\theta)+n \ln (c)-n \ln (1+\theta)+\sum_{i=1}^{n} \ln \left(1+x_{i}\right)+\exp \left(-\theta \sum_{i=1}^{n} x_{i}\right) \\
& +(c-1) \ln \left\{-\log \left(1-\left(1-\left(\left(\theta+1+\theta \sum_{i=1}^{n} x_{i}\right) /(\theta+1)\right) \exp \left(-\theta \sum_{i=1}^{n} x_{i}\right)\right)\right)\right\} \\
& -n(c-1) \ln (\beta)-n \ln (\beta)-\ln \left\{\sum_{i=1}^{n}\left(1-\left(1-\left(\left(\theta+1+\theta x_{i}\right) /(\theta+1)\right) \exp \left(-\theta x_{i}\right)\right)\right)\right\} \\
& +\left[-\frac{\log \left(1-\left(1-\left(\left(\theta+1+\theta \sum_{i=1}^{n} x_{i}\right) /(\theta+1)\right) \exp \left(-\theta \sum_{i=1}^{n} x_{i}\right)\right)\right)}{\beta}\right]^{c} .
\end{aligned}
$$

To obtain the normal equations, we take partial derivatives with respect to $\psi=(\theta, \beta, c)$ which results in the following equations after simplifications:

$$
\begin{aligned}
\frac{\mathrm{d} l}{\mathrm{~d} \theta}=0 \Longrightarrow & \frac{2 n}{\theta}+\sum_{i=1}^{n}\left(\theta-\frac{x_{i}}{\left(1+\theta+x_{i}\right)}\right)+(c-1) \sum_{i=1}^{n}\left(\frac{x_{i}}{\left(\theta+1+\theta x_{i}\right)}-\frac{1}{(\theta+1)}-x_{i}\right)-\frac{1}{\beta^{c}} \sum_{i=1}^{n}\left(\frac{c x_{i}}{\left(\theta+1+\theta x_{i}\right)}-c x_{i}-\frac{c}{(\theta+1)}\right) \\
\frac{\mathrm{d} l}{\mathrm{~d} c}=0 \Longrightarrow & \frac{n}{c}-n \ln (\beta)+\ln \left\{-\sum_{i=1}^{n} \frac{\log \left(\left(\left(\theta+1+\theta x_{i}\right) /(\theta+1)\right) \exp \left(-\theta x_{i}\right)\right)}{\beta}\right\}-\ln \left\{-\sum_{i=1}^{n} \frac{\log \left(\left(\left(\theta+1+\theta x_{i}\right) /(\theta+1)\right) \exp \left(-\theta x_{i}\right)\right)}{\beta}\right\} \\
\frac{\mathrm{d} l}{\mathrm{~d} \beta}=0 \Longrightarrow & \frac{-n c}{\beta}-(c-1) \beta^{-2} \ln \left\{-\sum_{i=1}^{n}\left(\log \left(\left(\left(\theta+1+\theta x_{i}\right) /(\theta+1)\right) \exp \left(-\theta x_{i}\right)\right)\right)\right\} \\
& +\frac{c}{\beta^{(c+1)}}\left\{-\sum_{i=1}^{n}\left(\log \left(\left(\left(\theta+1+\theta x_{i}\right) /(\theta+1)\right) \exp \left(-\theta x_{i}\right)\right)\right)\right\}^{c}
\end{aligned}
$$

Analytical solutions of the above normal equations may be difficult to obtain through the usual computation methods. Therefore, we suggest the use of embedded computer software such as R or MATLAB to obtain numerical solutions for the parameters.

\section{Simulation Study}

In this section, we demonstrate some results based on Monte Carlo simulations to compare the performance of the parameter estimates when the MLE method is applied. In our simulation study, we employed samples of sizes $n=10,20,30$, 40 , and 50, parameter combinations as $\beta=1, \theta=0.5,1.0$, and 3.0, and $c=1.0$ and 3.0 with 5000 iterations. Using the GWL distribution with the above sets of parameters, we generated random samples repeatedly 5000 times. Parameter estimates were computed as well as their biases and mean squared errors (MSEs). Finally, the average bias and average mean square error (MSE) of the parameter estimates were computed over the 5000 replications. Without loss of generality, $\beta$ is fixed as a unit to avoid convergence issues. Simulation outputs are displayed in Table 2. The following points are observed from Table 2. The average bias and average MSE decrease as the sample size increases for both parameters estimated in this study as expected. It can be further observed that both estimates are positively biased. The simulation results also show that there is no considerable difference in the average bias for different choices of the parameters.

\section{Application of Generalized Weibull-Lindley (GWL) in Modeling Real-Life Survival Data}

We applied this new generated distribution to model two real survival datasets. The first dataset is related to relief times (in minutes) of 20 patients receiving an analgesic as reported by Gross and Clark (1975, p. 105) [14]. The data are as follows: 1.1, 1.4, 1.3, 1.7, 1.9, 1.8, 1.6, 2.2, 1.7, 2.7, 4.1, 1.8, $1.5,1.2,1.4,3.0,1.7,2.3,1.6,2.0$. 
TABLe 2: Average biases and MSEs of the ML estimates of the parameters of GWL distribution.

\begin{tabular}{|c|c|c|c|c|c|c|}
\hline \multicolumn{3}{|c|}{ True values } & \multicolumn{2}{|c|}{ Bias } & \multicolumn{2}{|c|}{ MSE } \\
\hline$n$ & $c$ & $\theta$ & $\widehat{c}$ & $\hat{\theta}$ & $\widehat{c}$ & $\hat{\theta}$ \\
\hline 10 & 1 & 0.5 & 0.1641 & 0.0313 & 0.1447 & 0.0197 \\
\hline 20 & 1 & 0.5 & 0.0732 & 0.0171 & 0.0450 & 0.0083 \\
\hline 30 & 1 & 0.5 & 0.0481 & 0.0115 & 0.0264 & 0.0054 \\
\hline 40 & 1 & 0.5 & 0.0359 & 0.0081 & 0.0182 & 0.0038 \\
\hline 50 & 1 & 0.5 & 0.0289 & 0.0056 & 0.0142 & 0.0030 \\
\hline 100 & 1 & 0.5 & 0.0153 & 0.0030 & 0.0065 & 0.0014 \\
\hline 10 & 1 & 1.0 & 0.1633 & 0.0692 & 0.1448 & 0.0901 \\
\hline 20 & 1 & 1.0 & 0.0728 & 0.0373 & 0.0453 & 0.0373 \\
\hline 30 & 1 & 1.0 & 0.0480 & 0.0249 & 0.0266 & 0.0238 \\
\hline 40 & 1 & 1.0 & 0.0359 & 0.0177 & 0.0183 & 0.0170 \\
\hline 50 & 1 & 1.0 & 0.0290 & 0.0124 & 0.0143 & 0.0132 \\
\hline 100 & 1 & 1.0 & 0.0154 & 0.0064 & 0.0065 & 0.0063 \\
\hline 10 & 1 & 3.0 & 0.1640 & 0.2573 & 0.1465 & 1.1013 \\
\hline 20 & 1 & 3.0 & 0.0729 & 0.1358 & 0.0462 & 0.4370 \\
\hline 30 & 1 & 3.0 & 0.0481 & 0.0904 & 0.0272 & 0.2741 \\
\hline 40 & 1 & 3.0 & 0.0360 & 0.0642 & 0.0187 & 0.1942 \\
\hline 50 & 1 & 3.0 & 0.0291 & 0.0456 & 0.0146 & 0.1505 \\
\hline 100 & 1 & 3.0 & 0.0154 & 0.0237 & 0.0066 & 0.0716 \\
\hline 10 & 3 & 0.5 & 0.4956 & 0.0068 & 1.3236 & 0.0018 \\
\hline 20 & 3 & 0.5 & 0.2205 & 0.0040 & 0.4154 & 0.0008 \\
\hline 30 & 3 & 0.5 & 0.1449 & 0.0027 & 0.2442 & 0.0005 \\
\hline 40 & 3 & 0.5 & 0.1082 & 0.0019 & 0.1679 & 0.0004 \\
\hline 50 & 3 & 0.5 & 0.0873 & 0.0012 & 0.1314 & 0.0003 \\
\hline 100 & 3 & 0.5 & 0.0460 & 0.0007 & 0.0598 & 0.0002 \\
\hline 10 & 3 & 1.0 & 0.4948 & 0.0145 & 1.3254 & 0.0078 \\
\hline 20 & 3 & 1.0 & 0.2201 & 0.0085 & 0.4169 & 0.0036 \\
\hline 30 & 3 & 1.0 & 0.1446 & 0.0058 & 0.2453 & 0.0024 \\
\hline 40 & 3 & 1.0 & 0.1080 & 0.0040 & 0.1686 & 0.0017 \\
\hline 50 & 3 & 1.0 & 0.0872 & 0.0026 & 0.1320 & 0.0014 \\
\hline 100 & 3 & 1.0 & 0.0459 & 0.0014 & 0.0601 & 0.0007 \\
\hline 10 & 3 & 3.0 & 0.4942 & 0.0515 & 1.3306 & 0.0892 \\
\hline 20 & 3 & 3.0 & 0.2197 & 0.0298 & 0.4201 & 0.0411 \\
\hline 30 & 3 & 3.0 & 0.1443 & 0.0202 & 0.2473 & 0.0272 \\
\hline 40 & 3 & 3.0 & 0.1078 & 0.0142 & 0.1700 & 0.0198 \\
\hline 50 & 3 & 3.0 & 0.0871 & 0.0094 & 0.1332 & 0.0156 \\
\hline 100 & 3 & 3.0 & 0.0459 & 0.0051 & 0.0606 & 0.0077 \\
\hline
\end{tabular}

TABLE 3: Relief times in months of cancer patients from Lee and Wang [15].

$4.50,32.15,19.13,4.87,14.24,5.71,7.87,7.59,5.49,3.02,2.02,4.51,9.22,1.05,3.82,9.47,26.31,79.05,2.02,2.62,4.26,0.90,11.25,21.73,10.34$, $10.66,0.51,12.03,3.36,2.64,43.01,14.76,0.81,1.19,3.36,8.661 .46,14.83,5.62,18.10,17.14,25.74,15.96,17.36,1.35,4.33,9.02,22.69,6.94$, $2.46,7.26,3.48,4.23,3.70,6.54,3.64,8.65,3.57,5.41,11.64,2.09,2.23,6.25,7.93,4.34,25.82,12.02,3.88,13.80,5.85,7.09,20.28,5.32$, $46.12,5.17,2.8,0.20,8.37,36.66,14.77,10.06,8.53,4.98,11.98,5.06,1.76,16.62,4.40,12.07,34.26,6.97,2.07,0.08,17.12,1.40,12.63,2.75$, $7.66,7.32,4.18,1.26,13.29,6.76,23.63,3.25,7.62,7.63,3.52,2.87,9.74,3.31,0.40,2.26,5.41,2.69,2.54,11.79,2.69,5.34,8.26,6.93,0.50$, $10.75,5.32,13.11,5.09,7.39$.

The second dataset represents the remission times (in months) of a random sample of 128 cancer patients obtained from Lee and Wang (2003, p. 231) [15]. The data are shown in Table 3.

For comparison purposes, four additional models were also fitted including Weibull-Lindley (WL) [16], new Weibull-F [17], two-parameter Lindley (TPL) [18], Akash distribution [19], and Lindley distribution [9]. Comparisons were made using AIC, CAIC, BIC, HQIC, and minus loglikelihood values with the criteria of selecting the model with smaller values. Results are displayed in Tables 4 and 5 .
By examining Table 4, the GWL distribution provides the best fit for survival time of 20 patients receiving an analgesic as reported by Gross and Clark based on the values of log-likelihood, AIC, BIC, CAIC, and HQIC. Similarly, results displayed in Table 5 provide information about the superiority of GWL distribution in modeling remission time of cancer patients after receiving treatments based on the values of log-likelihood, AIC, BIC, CAIC, and HQIC which both are in favor to generalized Weibull-Lindley (GWL) distribution. 
TABLe 4: Analysis results of remission dataset by Gross and Clark [14].

\begin{tabular}{|c|c|c|c|c|c|c|}
\hline Distribution & Parameters & $-2 \log L$ & AIC & $\mathrm{BIC}$ & CAIC & HQIC \\
\hline GWL & $\beta=0.869, c=1.973, \theta=0.696$ & 21.382 & 48.764 & 51.751 & 50.264 & 49.347 \\
\hline Akash & $\delta=1.157$ & 59.500 & 61.700 & 62.500 & 61.700 & 60.645 \\
\hline Lindley & $\theta=0.814$ & 30.250 & 62.499 & 63.495 & 62.722 & 62.694 \\
\hline TPL & $\theta=1.110, \alpha=0.004$ & 26.258 & 56.518 & 58.509 & 57.224 & 56.907 \\
\hline WL & $\alpha=1.887, \lambda=0.964, \beta=0.024$ & 30.838 & 67.677 & 70.664 & 69.177 & 68.260 \\
\hline
\end{tabular}

TABle 5: Analysis results of remission dataset by Lee and Wang [15].

\begin{tabular}{lccccc}
\hline Distribution & Parameters & $-2 \log L$ & AIC & BIC & CAIC \\
\hline GWL & $\beta=\mathbf{1 . 4 8 9}, c=\mathbf{0 . 8 3 8}, \theta=\mathbf{0 . 2 7 8}$ & $\mathbf{4 1 2 . 6 5 5}$ & $\mathbf{8 3 1 . 3 0 9}$ & $\mathbf{8 3 9 . 8 6 5}$ & $\mathbf{8 3 1 . 5 0 3}$ \\
NW-F & $\alpha=5.355, \beta=0.222, \Theta=0.600$ & $\mathbf{4 0 9 . 3 8 7}$ & 831.520 & 840.080 & 831.720 \\
Lindley & $\theta=0.201$ & 448.225 & 841.201 & 844.0531 & 841.233 \\
TPL & $\theta=0.203, \alpha=1.466$ & 418.993 & 841.986 & 847.690 & 842.082 \\
WL & $\alpha=0.407, \lambda=0.143, \beta=1.470$ & 521.8221 & 1049.644 & 1058.2 & 835.000 \\
\hline
\end{tabular}

\section{Conclusion}

The new lifetime distribution named as generalized Weibull-Lindley distribution is developed in this article. Some statistical properties of the developed distribution are discussed. A simulation study is also given to learn the performance of the shape parameters. Two real datasets are employed to fit the proposed distribution, and the results demonstrate high performance for the newly established generalized Weibull-Lindley (GWL) distribution. Future research may be considered as the other versions of Weibull distribution or Lindley distribution or both to generate other forms of GWL distributions and several more lifetime datasets with complicated structure employed to evaluate their performance.

\section{Data Availability}

The used datasets are given in the manuscript.

\section{Conflicts of Interest}

The authors declare that they have no competing interests.

\section{Authors' Contributions}

Pius Marthin carried out methodology and computations and GS Rao contributed to writing and data collection. Both authors read and approved the final manuscript.

\section{References}

[1] A. Alzaghal, C. Lee, and F. Famoye, "Exponentiated T-X family of distributions with some applications," International Journal Probability and Statistics, vol. 2, pp. 31-49, 2013.

[2] A. Azzalini, "A class of distributions which includes the normal ones," Scandinavian Journal of Statistics, vol. 12, pp. 171-178, 1985.

[3] A. Azzalini and A. Capitanio, "Distributions generated by perturbation of symmetry with emphasis on a multivariate skew t-distribution," Journal of the Royal Statistical Society: Series B (Statistical Methodology), vol. 65, no. 2, pp. 367-389, 2003.
[4] A. Marshall and I. Olkin, "A new method for adding a parameter to a family of distributions with application to the exponential and Weibull families," Biometrika, vol. 84, no. 3, pp. 641-652, 1997.

[5] N. Eugene, C. Lee, and F. Famoye, "Beta-normal distribution and its applications," Communications in Statistics-Theory and Methods, vol. 31, no. 4, pp. 497-512, 2002.

[6] M. C. Jones, "Families of distributions arising from distributions of order statistics," Test, vol. 13, no. 1, pp. 1-43, 2004.

[7] G. M. Cordeiro and M. de Castro, "A new family of generalized distributions," Journal of Statistical Computation and Simulation, vol. 81, no. 7, pp. 883-898, 2011.

[8] A. Alzaatreh, C. Lee, and F. Famoye, "A new method for generating families of continuous distributions," Metron, vol. 71, no. 1, pp. 63-79, 2013.

[9] M. E. Ghitany, B. Atieh, and S. Nadarajah, "Lindley distribution and its application," Mathematics and Computers in Simulation, vol. 78, no. 4, pp. 493-506, 2008.

[10] P. R. D. Marinho, C. R. D. Diaz, and M. Bourguignon, "Adequacymodel. an $\mathrm{r}$ package for modeling probability distributions and general optimization," 2016, https:// www.r-project.org/.

[11] J. J. A. Moors, "A quantile alternative for kurtosis," The Statistician, vol. 37, no. 1, pp. 25-32, 1988.

[12] F. Galton, Enquiries into Human Faculty and its Development, Macmillan, London, UK, 1883.

[13] D. D. Hanagal, Modeling Survival Data Using Frailty Models, Chapman \& Hall, New York, NY, USA, 2011.

[14] A. J. Gross and V. A. Clark, Survival Distributions: Reliability Applications in the Biometrical Sciences, John Wiley, New York, NY, USA, 1975.

[15] E. T. Lee and W. Wang, Statistical Methods for Survival Data Analysis, John Wiley \& Sons, Hoboken, NJ, USA, 2003.

[16] T. G. Ieren, S. O. Oyamakin, and A. U. Chukwu, "Modeling lifetime data with Weibull-Lindley distribution," Biometrics \& Biostatistics International Journal, vol. 7, no. 6, pp. 532-544, 2018.

[17] Z. Ahmad, M. Elgarhy, and G. G. Hamedani, "A new Weibull$X$ family of distributions: properties, characterizations and applications," Journal of Statistical Distributions and Applications, vol. 5, no. 5, pp. 1-18, 2018.

[18] R. Shanker, K. K. Kamlesh, and H. A. Fesshaye, "Two parameter Lindley distribution: its properties and applications," Biostatistics and Biometrics Open Access Journal, vol. 1, no. 4, pp. 555-570, 2017. 
[19] R. Shanker and H. Fesshaye, "On modeling of lifetime data using Akash, shanker, Lindley and exponential distributions," Biometrics \& Biostatistics International Journal, vol. 3, no. 6, pp. 214-224, 2016. 